\title{
ENSAYO
}

\section{Modernidad, Modernismo y Tecnología: concepciones y valoraciones}

Por: M.A.Roxana Reyes Rivas', Instituto Tecnológico de Costa Rica

Fecha de recibido: $\quad 6$ de febrero, 2016.

Fecha de aceptación: 22 de mayo, 2016.

\section{Resumen}

Se describen y discuten el concepto de tecnología y las diferentes valoraciones que esta recibe en el pensamiento europeo desde los albores de la Modernidad hasta el siglo XX. Para ello no solamente se recurrirá al concepto de Modernidad, más conocido, sino que también se incluirá el concepto de modernismo como lo utiliza Scott Lash en su obra Sociología del posmodernismo (1997). Este no solo lo considera como un movimiento estético, sino como una experiencia histórica y social. Este recorrido empezará con la discusión de Descartes y Bacon en el siglo XVII, para continuar con la crítica de Marx y Engels en el siglo XIX, posteriormente, se examinarán los acercamientos a dicha época por parte de Foucault y Bell. Luego, se verá el abordaje sobre la tecnología practicado, en el siglo XX, por Horkheimer, Adorno, Marcuse y Benjamín. Por último, se ofrece una propuesta de acercamiento al asunto.

\section{Abstract}

\section{Modernity, Modernism and Technology: concepts and evaluations}

The concept of technology and the different appreciations which it receives in the European thought since the dawn of Modernity until the XX Century are described and discussed. In order to do so, the paper not only will resort to the better-known concept of Modernity, but it will also include the concept of modernism as used by Scott Lash in his work Sociología del posmodernismo (1997). This does not only consider it as an aesthetics movement, but also as a historical and social experience. This description will depart from the discussion of Descartes and Bacon in the XVII Century, in order to continue with MarX and Engels criticism in the XIX Century. Later the approaches at that time by Foucault and Bell will be studied. Finally, a look will be taken at the approach on technology practiced in the $\mathrm{XX}$ Century, by Horkheimer, Adorno, Marcus and Benjamín. Lastly, a proposal of an approach to the matter in discussion. is presented.

1 Roxana Reyes es máster en Filosofía. Labora como docente e investigadora del Instituto Tecnológico de Costa Rica y de la Universidad de Costa Rica. Contacto: roxana_reyes@ yahoo.com.
Roxana Reyes Rivas. Modernidad, Modernismo y Tecnología: concepciones y valoraciones. Revista Comunicación. Año 37 , volumen 25, número 2, julio - diciembre, 2016. Instituto Tecnológico de Costa Rica. ISSN: 0379-3974 / e-ISSN1659-3820.

\section{PALABRAS CLAVE:}

Modernidad, modernismo, Revolución científica, Revolución Industrial, razón instrumental, tecnología, utilidad y eficacia.

\section{KEY WORDS :}

Modernity, modernism, Scientific Revolution, Industrial Revolution, instrumental reason, technology, usefulness and efficacy. 


\section{INTRODUCCIÓN}

Hay muchas maneras de entender cuándo comienza la Modernidad, en tanto conjunto de conceptos e ideales que marcan un giro en la cultura de Occidente. Normalmente se entiende por este período al que empieza con la Revolución científica, por un lado y, por otro, la disolución del mundo medieval a partir del Renacimiento. Estos hechos marcan un giro en el pensamiento filosófico tanto acerca del conocimiento, como acerca de la política. A su vez, también marca un giro en otros ámbitos de la cultura como el arte y la literatura.

Si bien es erróneo pensar que el desarrollo de la inventiva humana que hoy conocemos como tecnología empezó con la Revolución científica, sí es verdadero que esta ha sido catapultada de una manera inusual a partir de aquella. En efecto, grandes desarrollos en la hidráulica pueden ser encontrados durante la Edad Media. Sin embargo, la profunda fascinación que la cultura europea va a experimentar, a partir del siglo XVII, con la fabricación de máquinas y artilugios -y sobretodo con su utilidad- no tiene parangón en la historia que la precedió.

En este trabajo me propongo hacer una discusión crítica del concepto de tecnología ${ }^{2}$ y su valoración en la Modernidad. Para esto me ocuparé primero de la valoración de la eficiencia y la utilidad en los albores de la Modernidad, valiéndome del discurso de pensadores como René Descartes y Francis Bacon. Posteriormente discutiré la influencia del desarrollo tecnológico durante la Revolución Industrial, en el movimiento cultural Ilamado modernismo, siguiendo para ello el concepto de modernismo usado por Lash en Sociología del posmodernismo (1997). Al dar cuenta de estos dos momentos del pensamiento sobre la tecnología, intentaré mencionar voces escépticas acerca de las bondades de la tecnología moderna tanto en el mismo siglo XIX (Marx y Engels) como sobre este período, en el caso de Michel Foucault y Daniel Bell. También discutiré la crítica elaborada durante el siglo XX al respecto. Para ello mencionaré a autores como Theodor Adorno, Max Horkheimer, Herbert Marcuse y Walter Benjamin. Para terminar con este trabajo ofreceré una reflexión sobre la manera en que debería abordarse el examen y valoración de la tecnología.

2 En nuestros días, normalmente se entiende tecnología como "... dominios "avanzados" para los cuales el soporte científico de la creación tecnológica se encuentran extremadamente desarrollados" (Daumas 1996, p. 8).

\section{MODERNIDAD Y TECNOLOGÍA}

\author{
Tell someone that you are reading \\ about the history of science \\ and their first reaction will be to ask: "What's that?" \\ We instinctively associate science \\ with the modern world, not with past. \\ Peter J. Bowler and Iwan Rhys Morus \\ Making Modern Science
}

Es una creencia muy extendida que la revolución copernicana, iniciada en el siglo XVI, es una de las grandes inspiraciones del gran vuelco en el pensamiento y la cultura europeos que conocemos hoy como Modernidad. (Dear, 2001, p. 37 y Belaval, 1976, p. 4 y ss.) Es cierto que una propuesta diferente en la forma de buscar y evaluar el conocimiento significó un golpe de gracia no solamente a formas tradicionales de juzgarlo, sino de elaborar filosofía. Sin embargo, la verdad es que posiblemente otros eventos y cambios paulatinos que se venían operando desde antes contribuyeron tanto como Copérnico en la revolución registrada en la cultura occidental de entonces.

En efecto, la Modernidad está marcada por formas de hacer filosofía que se ocupan tanto de examinar las condiciones del conocimiento verdadero, como por un rechazo a las formas antiguas por considerarlas inútiles. Esta consideración sobre la filosofía y el conocimiento medieval como inútiles tiene dos aristas interesantes. Por una parte, estos carecen de utilidad porque no permiten comprender el mundo que nos rodea. Por otra parte, no facilitan la creación de máquinas y objetos que sean capaces de facilitar la vida humana.

Así Descartes (1596-1650) y Bacon (1561-1626) en sus obras se quejarán de la poca utilidad de ramas de conocimiento como la lógica y la filosofía aristotélicas. Para Bacon en The New Organon (1620/1960), la ciencia es útil porque permite dominar la naturaleza (Libro I, af. III). Para él la ciencia es una especie de técnica (Libro I, af. II). De hecho, su crítica a la ciencia medieval (aristotélica) es que esta no permite producir obras de utilidad:

Más aun, las obras ya conocidas se deben más a la casualidad y al experimento que a las ciencias; pues las ciencias que hoy poseemos son simplemente sistemas de ordenamiento agradable y exposición de cosas ya inventadas, 
no métodos de invención o instrucciones para nuevas obras." (Libro I, af. VIII, trad. RRR).

$\mathrm{Y}$, a pesar de que pertenece a una tradición filosófica diferente, Descartes se referirá en términos muy parecidos en el Discurso del método. Para él mediante estudio de la ciencia ha logrado encontrar cosas que serán de mucha utilidad que también nos haga "dueños y poseedores de la naturaleza" e inclusive puede ayudarnos a buscar "medios para que el hombre sea más sabio e ingenioso" (Descartes, 1945).

Lo interesante de ambos autores es que están pensando en que el pensamiento científico debe ser un recurso para crear algo más allá de lo que la naturaleza nos brinda. Es evidente que aunque el siglo XVII no es uno en que se haya creado una gran tecnología, estos pensadores podían vislumbrar las posibilidades que esta nueva ciencia ofrecía. Ahora bien, como mencioné arriba, las máquinas ${ }^{3}$ son objetos que se conocen con mucha anterioridad a la ciencia a que nos estamos refiriendo. No obstante, esa relación que hoy día se considera fundante de la tecnología empieza a gestarse en los inicios de la Modernidad.

Ahora, como anoté previamente, que posiblemente desde el punto de vista de la cultura europea a que me estoy refiriendo, hubo otros eventos que propiciaron tanto como la revolución científica el advenimiento de la tecnología que desembocaría en la revolución industrial. Lewis Mumford (1895-1990) en su clásica obra Técnica y civilización (1934) describe una serie de hechos y procesos que fueron preparando el terreno en que finalmente floreció la tecnología moderna. En efecto, de acuerdo con este autor, tan importante es la invención del reloj para regular la rutina del monasterio benedictino (siglo VII) o el interés de la alquimia y la magia en manipular y transformar el mundo, como el orden burgués y los viajes de exploración, para comprender el caldo de cultivo de esta tecnología.

Así pues, el reloj que permite regimentar la vida del monasterio, también sirve para concebir el tiempo

3 Soy consciente de que el concepto de tecnología no necesariamente se reduce al de máquina, pero la menciono aquí pues esta es uno de los objetos que más asociamos con la tecnología y posiblemente es la cara más visible de la tecnología. Hoy las máquinas que han sido creadas a través de la historia de Occidente están comprendidas dentro del gran complejo tecnológico. Por complejo tecnológico se entiende "el conocimiento, las pericias y las artes derivadas de la industria o implicadas en la nueva técni$\mathrm{ca}$, e incluirá varias formas de herramientas, instrumentos, aparatos y obras así como máquinas propiamente dichas" (Mumford, 1998, p. 29). como algo independiente de los ritmos orgánicos. Transformase así el mundo en un conjunto de secuencias matemáticamente mensurables. En palabras de Mumford:

El reloj, no la máquina de vapor, es la máquina clave de la moderna edad industrial.(...) En su relación con cantidades determinables de energía, con la estandarización, con la acción automática, y finalmente con su propio producto especial, el tiempo exacto, el reloj ha sido la máquina principal en la técnica moderna: y en cada período ha seguido a la cabeza: marca una perfección hacia la cual aspiran otras máquinas (1998, p. 31).

Por otra parte, entre los siglos XIV y XVI el espacio también se convierte en un sistema mensurable, como lo demuestra el manejo de la perspectiva:

Los cuerpos no existían separadamente como magnitudes absolutas: estaban coordinadas con otros cuerpos dentro del mismo marco de la visión y debían estar a escala (Mumford, 1998, p. 36).

Así pues, la mensurabilidad y su adopción por el capitalismo naciente son anteriores a la revolución científica y constituyen, junto con esta, rasgos típicos de la Modernidad. Lo que sí es cierto es que, tanto en lo que se refiere a la nueva ciencia, como la objetivación del tiempo y el espacio, el énfasis en la matematización fueron fundamentales para el desarrollo posterior de la Modernidad y sus rasgos más característicos.

Mi interés en mencionar todos estos aspectos es que se pueda ver cómo no solamente hay una apreciación diferente de la importancia del conocimiento, sino que hay una serie de condiciones que se han ido gestando en diferentes momentos anteriores o muy tempranos de la Modernidad que permiten el desarrollo de la tecnología moderna. El desarrollo de la ciencia moderna no solamente supone un cambio de métodos y finalidades, sino que propone como rasgos fundamentales de sí la utilidad y la eficacia. Es decir, la capacidad de manipular y transformar el entorno en interés de obtener beneficios y la exigencia de obtener resultados prácticos a partir del conocimiento, no llevan a la Modernidad más que a una cultura de gran culto de estas mismas características en la vida cotidiana, posibilitada por la máquina.

De esta forma, el siglo XVIII se encuentra no solamente con las condiciones científico -técnicas para catapultar 
toda la oleada de inventiva que conoceremos como la Revolución Industrial, sino que aquellos que ponen su ingenio al servicio de la nueva técnica, se encuentran totalmente respaldados por un nuevo culto a la eficiencia y la utilidad representadas por la máquina u otros inventos de la época. En otras palabras, los nuevos inventos, en tanto que representantes de la utilidad y la eficacia que se puede alcanzar mediante la ciencia, son valorados como algo bueno y deseable.

No obstante, muchas veces los procesos, productos o máquinas que se creaban no eran necesariamente mejores que aquello con lo que se contaba anteriormente. Lo importante es, pues, que la episteme del siglo XVIII alberga en sí el imperativo de inventar. Las nuevas invenciones son percibidas como buenas, sobretodo la máquina, pues ¿qué es la máquina sino el ideal de la dedicación al trabajo tan cara al mundo burgués? La fe es puesta ahora en la tecnología, ya que con las prestaciones de esta y con los descubrimientos de la nueva ciencia, la fe y el motor de la vida se alejan cada vez más de la religión. (Mumford, 1998, p. 72)

Empero, ya para finales del siglo XVIII empiezan a levantarse las primeras voces en contra de las consecuencias de la explosión técnica que ha tenido lugar durante esa época. En efecto, no bien ha empezado la revolución industrial cuando se ven también los primeros horrores provocados por el culto a la técnica, a saber, las condiciones espantosas de trabajo en las fábricas de entonces. William Blake (1757-1827) escribirá:

Es cosa santa el ver/ En tierra rica y fructífera/ Los niños reducidos a la miseria,/ Por mano fría y avara alimentados./ ¿Es una canción ese grito trémulo?/ ¿Podrá ser una canción de gozo?/ ¿Cuántos niños pobres hay?/ ¿Es esta la tierra de la pobreza? (citado por Cernuda, 1997, p. 15).

En resumen, en los textos y hechos que he discutido se encuentran preconizados los conceptos de utilidad y eficacia como prestaciones de la ciencia que se traducirán en máquinas y procedimientos que posteriormente conoceremos como tecnología ${ }^{4}$. En otras palabras, el concepto de un tipo diferente de actividad ligada a la nueva ciencia y que supone la posibilidad de transformar el entorno y extraer riqueza de ello, posteriormente se denotará con el término "tecnología". Pero esta no hubiera podido desarrollarse y florecer con la fuerza que

4 De acuerdo con Maurice Daumas (1996) el término "tecnología" se acuña en el siglo XVII. lo hizo, si la valoración alrededor de ella no hubiera sido la de una gran esperanza y bondad.

Para finales del siglo XVIII la valoración optimista de los productos de la razón (conocimiento y técnica) ha arraigado en la vida intelectual de los entonces ideales de la llustración. Esto no impide que, posteriormente, con el advenimiento de la Revolución Industrial, se hayan visto rápidamente algunas de las peores facetas generadas por esta nueva tecnología, contra ella se revelará Blake y posteriormente Marx y Engels, a quienes me referiré más adelante.

\section{MODERNISMO Y TECNOLOGÍA}

Dado que mi discusión se centra tanto en el concepto de tecnología, como en el de su valoración, he preferido usar el término modernismo en vez de Modernidad en el sentido en que lo usa Lash en su obra Sociología del posmodernismo (1997) $)^{5}$. Creo que esta categoría es muy enriquecedora para este trabajo, pues permite ver mejor no solamente el concepto de tecnología, sino sus valoraciones. En efecto, este autor entiende el modernismo no solamente como un giro en el arte a finales del siglo XIX, sino que sostendrá:

...que el modernismo estético y sus correlatos sociales deben comprenderse como una transformación fundamental de este proyecto, que incluye no sólo la profundización y un socavamiento de la racionalidad de la llustración, sino también la trasmutación y el renovado desarrollo de la racionalidad instrumental (1997, p. 166).

En efecto, durante el siglo XIX asistimos a un gran desarrollo de la tecnología y el capitalismo. Si bien estos dos pueden ser vistos como fenómenos independientes, en la época que estoy examinando ambos procesos se intensifican y se retroalimentan mutuamente. La tecnología, sin duda, es el corazón mismo de la industrialización del siglo XIX, la cual es fomentada y aprovechada en grado sumo por los grandes poseedores de recursos financieros. En efecto, estos últimos fueron los que mejor usaron la tecnología en aras del beneficio privado, ya que eran quienes podían invertir en máquinas, procedimientos e instalaciones más

5 Lash diferencia su concepto del modernismo estético mediante un recurso tipológico, esto es, escribe "modernismo", a lo largo de este trabajo estaré usando el término "modernismo" en el sentido de Lash. 
eficientes que, a su vez, dinamizaran la producción de capital.

Surgen en este contexto el ingeniero y el inventor los cuales, cada vez más, dependerán del conocimiento de la ciencia contemporánea. Así, por ejemplo, si bien Thomas Newcomen (el inventor de la máquina de vapor, 16631729) era un herrero ingenioso, James Watt ${ }^{6}$ (1736-1819) fue un hombre con formación académica en matemática e ingeniería (Lilley, 1993, pp. 140-141). Lo interesante es que estas personas ya no se ocupan directamente de la ciencia como una explicación del mundo circundante, en particular de la naturaleza, como los filósofos naturales del XVII y XVIII. Más bien se dedican expresamente a la creación de máquinas, artefactos y procedimientos que mejoren -optimicen diríamos hoy- la utilidad del conocimiento. Esa utilidad y eficacia de la tecnología decimonónica no solamente no se traduce estrictamente en "resolución de problemas para facilitar la vida", sino que también se traduce en producción de beneficios y control.

Así, se profundiza, a mi entender, un rasgo que ya estaba de forma embrionaria en los valores de utilidad y eficacia de la racionalidad de la Modernidad, a saber, la razón instrumental. Lo interesante de la razón instrumental es que es una razón cada vez más autoreferencial en cuanto a sus propios valores de utilidad y eficacia. Es decir, se ocupa únicamente de alcanzar y medir sus metas en estos mismos términos. Pero esta autorreferencialidad también estaba en ciernes en el pensamiento tanto racionalista como empirista del XVII. Tanto Bacon como Descartes aconsejan solamente ocuparse del conocimiento útil, pero no involucrarse en política ni en polémicas religiosas. Por otra parte, muy pronto se unirá a esos valores, el de la eficiencia, que apunta a la rapidez con se pueden alcanzar resultados.

Pero esta razón instrumental no solamente tiene que ver con la inventiva industrial y la fabricación a gran escala. También esta se impone en la forma de regimentar la vida cotidiana: la puntualidad, las diferentes instituciones que controlan todo aquello que no está al servicio de los valores de la tecnología, a saber, la enfermedad física y mental, la vida de quienes no están directamente relacionados con la decencia burguesa como quienes son definidos como delincuentes o en prostitución. Es decir,

6 Watt fue el inventor del medidor de presión durante un ciclo de trabajo de una máquina de vapor y perfeccionador de esta. esta racionalidad se transforma en un instrumento de examen del ser humano mismo (Foucault, 1996).

Así pues, lo que acabo de discutir no parece más que una profundización de los ideales acuñados en la llustración dieciochesca con su culto a una racionalidad que, supuestamente, nos hará libres (Kant, 1784). En el XIX es un siglo burgués donde el culto al trabajo, la contabilización escrupulosa de gastos, beneficios y pérdidas, la medición del tiempo y la especialización de diferentes ramas de control social (medicina, psiquiatría, trabajo social, etc.) intentan imitar la eficiencia y precisión de una máquina. De ahí pues, el gran aprecio de la tecnología, sobretodo de la máquina en este siglo.

Sin embargo, la fábrica decimonónica es una de las grandes vergüenzas de la historia europea, como lo constata con vehemencia el poema de Blake que cité arriba. De esta forma la valoración de la tecnología entra en crisis en otros ámbitos, pues se la percibe como el instrumento de la opresión. Así pues, el modernismo estético es una reacción al constreñimiento del individuo por medio de una racionalidad que más bien anula lo humano y se convierte en un fin en sí misma o en un instrumento de control. En otras palabras, el modernismo en el arte y la literatura de finales del siglo XIX puede ser visto como una manifestación de un socavamiento de los ideales ilustrados de los que parece estar tan orgullosa la sociedad de su tiempo.

En este sentido podemos afirmar que el socavamiento de los ideales ilustrados proviene de la misma razón instrumental, hija del mismo racionalismo que inspiró la Ilustración, pero que deja poco espacio para la mayoría de edad que tanto promovían Kant y sus contemporáneos. De esta manera se explica el rechazo de estos artistas al orden impuesto por la racionalidad ilustrada en el arte, por ejemplo con respecto al uso de la perspectiva. De ahí lo que Daniel Bell ha denominado "ira contra el orden social" (1976, p. 13).

Un caso interesante del modernismo es el de Karl Marx (1818-1883) y Friedrich Engels (1820-1895). En el Manifiesto del Partido Comunista (1848) Marx y Engels ofrecen una explicación racional a la situación social de su época. En efecto, el Manifiesto... intenta proponer una forma de conocimiento para la realidad social que, desde su perspectiva no solo es cognoscible, sino dinámica y modificable. Pero, entendida a la luz de las "Tesis sobre 
Feuerbach" $(1845)^{7}$, la imagen de la realidad social se aparece como producto de la actividad del sujeto cognoscente.

En otras palabras, la realidad no es algo que afecta al sujeto cognoscente como si fuera algo separado de este, ni el sujeto constituye al objeto, por el contrario, ambos se constituyen mutuamente en la praxis. En este sentido podríamos decir que Marx y Engels siguen el principio de inmanencia característico del modernismo (Lash, 1997, p. 172) opuesto al dualismo sujeto-objeto de la llustración.

Así pues, bajo esta óptica no es posible proponer esencias, ni sustancias a la manera cartesiana, distinguiéndose el marxismo de otras propuestas epistémicas anteriores dentro de la Modernidad. Además, todo esto se basa en una concepción materialista dialéctica de inspiración hegeliana donde la realidad se entiende como una constante lucha entre contrarios y producto de la praxis. De esta forma se podría considerar al marxismo como una forma de pensamiento propia del modernismo.

Ahora bien, dentro de esta nueva propuesta epistémica se encuentra una realidad social que debe entenderse como lucha de clases (Marx y Engels, 1976, p. 30). De esta lucha de clases en la Modernidad encontramos a dos clases en pugna: burguesía y proletariado. Pero ¿qué es lo que mantiene a estas dos clases en pugna? Precisamente la posesión de unos y la desposesión de otros. Los burgueses mantienen su condición de poseedores mediante la constante innovación que produce más capital, es decir, en términos contemporáneos, esta clase se mantiene vigente mediante la introducción sostenida de tecnología:

La burguesía no puede existir sino a condición de revolucionar incesantemente los instrumentos de producción y, por consiguiente, las relaciones de producción y con ello todas las relaciones sociales (Marx y Engels, 1976, p. 33).

Esta cita tiene una arista muy interesante para el análisis que estoy ensayando en este trabajo. Esto es, la tecnología es, en gran parte, la que mantiene a la burguesía en el poder, de tal forma que, en última instancia, la lucha de clases es una lucha por la propiedad de la tecnología y los recursos financieros que la hacen posible. De esta forma el Manifiesto... se convierte en un hito en el análisis social de la tecnología y sus implicaciones.

$7 \quad$ Estoy consciente de que las "Tesis sobre Feuerbach" no fueron publicadas hasta 1888 , sin embargo consigno la fecha en que fueron redactadas por Marx para ver mejor la influencia que pudieron tener en el Manifiesto...
Más aun, de acuerdo con las predicciones del Manifiesto..., el capitalismo y la burguesía con él estaban destinados a perecer muy pronto, gracias al desarrollo tecnológico.

El progreso de la industria, del que la burguesía, incapaz de oponérsele, es agente involuntario, sustituye el aislamiento de los obreros, resultante de la competencia, por su unión revolucionaria mediante la asociación. (...) La burguesía produce, ante todo, sus propios sepultureros. Su hundimiento y la victoria del proletariado son igualmenteinevitables (MarxyEngels, 1976, p.43).

Desafortunadamente, Marxy Engels no pudieron pensaren su tiempo sobre otra clase o grupo revolucionario distinta al proletariado. En efecto, debido a que la burguesía no puede existir sin el proletariado, este último no puede desaparecer como sí desaparecerán otras clases. No será hasta muy avanzado el siglo XX que Herbert Marcuse (1898-1979) relocalizará al sujeto revolucionario. De eso me ocuparé en la siguiente sección.

\section{SIGLO XX Y TECNOLOGÍA}

El siglo XX ya no es solamente un siglo de grandes instalaciones manufactureras, industria pesada $y$ transporte por ferrocarril, sino que es también una época donde la tecnología atraviesa de manera más invasiva casi la totalidad de la experiencia humana. En efecto, el hecho de que mucha de la tecnología más visible sea aquella ligada al entretenimiento y otras formas de cultura, la hace no solo más presente en la vida cotidiana, sino que también la hace más transparente en tanto que llega el momento en que esta se naturaliza en nuestras vidas ${ }^{8}$.

Así pues, Max Horkheimer (1895-1973) y Theodor Adorno (1903-1969) en su obra Dialéctica del Iluminismo (1947) acuñaron el término "industrias culturales" para mencionar el fenómeno del uso de un criterio industrial más allá de la manufactura de productos materiales de uso básico. Es decir, la tecnología no solamente se usa para producir bienes materiales de consumo, maquinaria pesada o medios de transporte, sino que también se utiliza para difundir información y contenidos ideológicos que no siempre van en el mejor interés del público que los recibe. De hecho, más bien disminuye la capacidad

8 Solamente pensemos en las personas que mecánicamente encienden el televisor, la radio o la computadora apenas llegan a sus casas o se despiertan por la mañana. Muchas de ellas reportan que ni siquiera se dan cuenta de que lo han hecho. 
crítica de las personas y estimula un consumo desmedido confundible con la felicidad.

De acuerdo con estos autores las industrias culturales son una especie de degeneración del espíritu de la llustración. En otras palabras, toda aquella racionalidad que prometía convertirnos en mayores de edad doscientos años atrás, se ha convertido en razón instrumental sin cuestionarse por las consecuencias de sus productos. Por el contrario, esta razón instrumental dentro de las industrias culturales es capaz de mantenernos en minoría de edad incesantemente. Aun hoy el consumidor o la consumidora actuales reciben estímulos como infantes que deben contar constantemente con una gratificación inmediata.

También en esta misma línea encontramos a Walter Benjamin (1892-1940). En su opúsculo La obra de arte en la época de su reproductibilidad técnica (1936), critica la conversión de la obra de arte en un artículo barato y degradado al ser reproducido masivamente. Más aun, el arte tradicional, como la pintura empieza a imitar el arte comercial, se convierte en "centro de escándalo" (p. 16). Sin duda hablar del arte aurático de su culto supone una serie de valores emparentados con la Ilustración. Es decir, al parecer tanto Horkheimer y Adorno, como Benjamin piensan que hay que recuperar los ideales de la Ilustración, pero al hacerlo, por lo menos en una primera leída, proponen una solución elitista. Con respecto al manifiesto de Marinetti sobre la guerra colonialista contra Etiopía, Benjamin dirá, como si él supiera cuándo estará la sociedad lo suficientemente madura:

Este manifiesto tiene la ventaja de ser claro. Mereceque el dialéctico adopte su planteamiento de la cuestión. La estética de la guerra actual se le presenta de la manera siguiente: mientras que el orden de la propiedad impideel aprovechamiento natural de las fuerzas productivas, el crecimiento de los medios técnicos, de los ritmos, de la (Sic) fuentes de energía, urge un aprovechamiento antinatural. $Y$ lo encuentra en la guerra que, con sus destrucciones, proporciona la prueba de que la sociedad no estaba todavía lo bastante madura para hacer de la técnica su órgano, y de que la técnica tampoco estaba suficientemente elaborada para dominar las fuerzas elementales de la sociedad (Benjamín, 1973, p. 16).

Herbert Marcuse en su conocida obra El hombre unidimensional (1964) expresa también un gran desencanto con respecto de las sociedades altamente industrializadas. Para él, toda la comodidad y abundancia provista por la tecnología de mediados de siglo XX, es mayoritariamente un subterfugio para mantener a los seres humanos en la alienación y evitar así todo posible cuestionamiento o intento de cambio. Las formas de embrutecimiento que mantienen a los habitantes de las sociedades industrializadas se expresan en tecnologías muy sofisticadas. A la cabeza de los individuos, se encuentran los medios de comunicación masiva y el estímulo al consumo. Esto ha dado como resultado la unidimensionalidad en el pensamiento:

Los medios de transporte y comunicación de masas, los bienes de vivienda, (...) el irresistible rendimiento de la industria de diversiones y de la información, llevan consigo hábitos y actitudes prescritas, (...) que vinculan de forma más o menos agradable los consumidores a los productores y, a través de éstos, a la totalidad. (...) Así surge el modelo de pensamiento y conducta unidimensional en el que ideas, aspiraciones y objetivos, que trascienden por su contenido el universo establecido del discurso y la acción son rechazados o reducidos a los términos de este universo (Marcuse, 1964, p. 42).

Así pues, en estos autores hay una gran decepción de cómo las prestaciones de la tecnología contemporánea se alejan cada vez más de los intereses de las mayorías y, más bien, se vuelven instrumentos de dominación al servicio de intereses espurios.

Marcuse (1964), al igual que Horkheimer y Adorno (19471988), escribe su obra posterior a la Segunda Guerra Mundial, un momento de pérdida definitiva de la inocencia que aún podría haber quedado. Esto no solamente por el despliegue terrorífico de la razón instrumental en los campos de concentración nazis, sino por la capacidad destructiva desatada en grandes complejos tecnológicos como el Proyecto Manhattan. Pero Marcuse (1964) sí vislumbra nuevos sujetos revolucionarios posibles para revertir el carácter manipulador y amenazante de la tecnología contemporánea. Él ve en los jóvenes, los grupos étnicos discriminados, las mujeres y el lumpemproletariado la posibilidad de una rebelión a través de la imaginación, aunque sin dejar de pensar en usos de la tecnología más liberadores (Ibid, p. 280).

Pero no todos los pensadores que se han ocupado de la tecnología en el siglo XX tienen una visión tan sombría de 
esta. En su obra ya citada, Mumford (1998) considerará que todo intento racional por dominar la naturaleza, que cuaje en una tecnología, ha significado un gran avance en las sociedades desde el neolítico. Por supuesto, este autor escribió su obra en 1934 y se ocupa más del desarrollo de los ingenios tecnológicos de la Modernidad sin ocuparse de sus relaciones e implicaciones con otros ámbitos de la cultura como el arte o la literatura. En otras palabras, su trabajo es más inmanente.

No quiero terminar esta sección sin mencionar que con todas las posibles desventajas o perjuicios de la tecnología, con toda la manipulación ideológica para la que se presta, esta sigue siendo tenida en alta estima y se considera uno de los rasgos más distintivos de nuestra época. Por eso mismo ha dado pie inclusive a la inauguración de disciplinas académicas como los estudios sociales de la ciencia y la tecnología.

\section{CONCLUSIONES}

Se ha examinado y mostrado cómo se ha concebido la tecnología y las oscilaciones que ha tenido en su valoración a lo largo de diferentes momentos de la Modernidad. Para esto ha sido de gran utilidad el concepto de modernismo propuesto por Lash (1997). En efecto, aplicado al fenómeno de la tecnología permite ver las contradicciones y las profundizaciones que engendra el ideal tecnológico de la Modernidad al interior de esa misma Modernidad. En otras palabras, el modernismo no es un abandono de la Modernidad, sino que expresa un momento de la Modernidad donde esta se expresa en todas sus posibilidades.

En lo que se refiere al siglo XX, por razones de espacio solamente se pudo abarcar algunos pensadores sobretodo de la primera mitad de éste y, con excepción de Benjamin (1936), en la posguerra. Esta época es importante porque, por una parte, obedece a una intensificación en el uso de la tecnología mediática que empieza en el período de entreguerras $y$, por otra parte, en otros ámbitos de producción de tecnología ha quedado patente que la razón instrumental sin diálogo con la razón sustantiva puede llevar a la catástrofe. En otras palabras, la tecnología no es un ámbito humano neutral, ni químicamente puro para que pueda ser abandonado a su autorreferencialidad.

No obstante, la tecnología sigue conservando un estatus casi mítico en la cultura contemporánea. No solamente es un negocio lucrativo, sino que crea significado y subjetividades, sobre todo en lo que concierne a las tecnologías de la información y la comunicación. De esta forma se ofrece a la reflexión no en tanto en el quehacer que tiene algunas implicaciones o impactos en otros ámbitos de la sociedad, sino como una dimensión más de la sociedad y su cultura, que debe ser tomada en cuenta cuando se habla de estas últimas.

Sin embargo, el ser considerada una dimensión más de la sociedad y la cultura no significa que no deba pasar por un cuestionamiento. En otras palabras, no se puede incurrir en la falacia naturalista de pensar que porque deseamos y apreciamos lo útil, eso debe quedarse entre nosotros. Esto es, el argumento de que lo que "es asi" por consiguiente "debe ser asi", es un argumento inválido y peligroso cuando se aplica a la tecnología. No todo lo que tenemos en tecnología es lo deseable y es fundamental recuperar la capacidad de elegir sobre ellas y controlarlas. En nuestros días la búsqueda de participación en las decisiones sobre la adopción de tecnologías o el control de su uso debe ser una de las áreas en que más se debe ocupar la ciudadanía. Las comisiones de bioética y los movimientos ecologistas, entre otros, son parte de esta nueva forma de participación.

\section{REFERENCIAS BIBLIOGRÁFICAS}

Bacon, F. (1620/1960). The New Organon and Related Writings. New York: Liberal Arts Press.

Belaval, Y. (ed.) (1976). Racionalismo, empirismo, ilustración. México: Siglo Veintiuno Editores, S.A.

Benjamín, W. (1973). La obra de arte en la época de su reproductibilidad técnica. Madrid: Editorial Taurus.

Bell, D. (1976). Las contradicciones culturales del capitalismo. Descargado el 5 de octubre de 2008, desde http://perso.gratisweb.com/carlosmanzano/ Danielbell.htm

Bowler, P. J. e Iwan R. M. (2005). Making Modern Science. A Historical Survey. Chicago: The University of Chicago Press.

Cernuda, L. (1997) Prólogo. En: Blake, W. Matrimonio del Cielo y el Infierno, Los cantos de Inocencia y Los cantos de Experiencia. Madrid: Visor Libros.

Daumas, M. (1996). Las grandes etapas del progreso técnico. México: Fondo de Cultura Económica.

Dear, P. (2001). Revolutionizing the Sciences. European Knowledge and Its Ambitions, 1500-1700. New Jersey: Princeton University Press. 
Descartes, R. (1945). Discurso del método. Buenos Aires: Editora Espasa-Calpe, S.A.

Foucault, M. (1996). Las palabras y las cosas. México: Siglo XXI Editores.

Horkheimer, M. y Theodor A. (1947/1988). La industria cultural. Iluminismo como mistificación de masas. En: Dialéctica del Iluminismo. Buenos Aires: Sudamericana.

Kant, I. (1784). Constestación a la pregunta: ¿Qué es la Ilustración? En: Aramayo, R. (ed.) ¿Qué es la Ilustración? Madrid: Alianza Editorial.

Lash, S. (1997). Sociología del posmodernismo. Buenos Aires: Amorrortu.

Lilley, S. (1993). El progreso tecnológico de la Revolución Industrial, 1700-1914. En: Zamora A. y Alfaro, M. (Comp.) Dédalo y su estirpe. (Historia-Tecnología-
Filosofía). Cartago: Editorial Tecnológica de Costa Rica.

Marcuse, H. (1972). El hombre unidimensional. Traducción de Antonio Elorza. Novena edición. Barcelona: Editorial Seix Barral.

Marx, K. y Engels, F. (1976). Manifiesto del Partido Comunista. Traducción al español, Editorial Progreso. Moscú: Editorial Progreso.

Marx, K. (1970). Tesis sobre Feuerbach. En: Marx, K. y Engels, F. Tesis sobre Feuerbach y otros escritos filosóficos. Tomado de Obras escogidas de Carlos Marx y Federico Engels de Ediciones de Lenguas Extranjeras, Moscú, 1951. México: Editorial Grijalbo, S.A.

Mumford, L. (1998). Técnica y civilización. Madrid: Alianza Editorial. 